\title{
Review Article \\ Sphingolipids in High Fat Diet and Obesity-Related Diseases
}

\author{
Songhwa Choi ${ }^{1,2}$ and Ashley J. Snider ${ }^{1,2,3}$ \\ ${ }^{1}$ Department of Medicine and Molecular and Cellular Biology, Stony Brook University, Stony Brook, NY 11794, USA \\ ${ }^{2}$ Stony Brook Cancer Center, Stony Brook University, Stony Brook, NY 11794, USA \\ ${ }^{3}$ Northport VA Medical Center, Northport, NY 11768, USA
}

Correspondence should be addressed to Ashley J. Snider; ashley.snider@stonybrookmedicine.edu

Received 14 August 2015; Accepted 18 October 2015

Academic Editor: Marc Pouliot

Copyright (C) 2015 S. Choi and A. J. Snider. This is an open access article distributed under the Creative Commons Attribution License, which permits unrestricted use, distribution, and reproduction in any medium, provided the original work is properly cited.

\begin{abstract}
Nutrient oversupply associated with a high fat diet (HFD) significantly alters cellular metabolism, and specifically including sphingolipid metabolism. Sphingolipids are emerging as bioactive lipids that play key roles in regulating functions, in addition to their traditional roles as membrane structure. HFD enhances de novo sphingolipid synthesis and turnover of sphingolipids via the salvage pathway, resulting in the generation of ceramide, and more specifically long chain ceramide species. Additionally, HFD elevates sphingomyelin and sphingosine-1 phosphate (S1P) levels in several tissues including liver, skeletal muscle, adipose tissue, and cardiovascular tissues. HFD-stimulated sphingolipid generation contributes to systemic insulin resistance, dysregulated lipid accumulation, and cytokine expression and secretion from skeletal muscle and adipose tissues, exacerbating obesity-related conditions. Furthermore, altered sphingolipid levels, particularly ceramide and sphingomyelin, are involved in obesity-induced endothelial dysfunction and atherosclerosis. In this review, HFD-mediated sphingolipid metabolism and its impact on HFDinduced biology and pathobiology will be discussed.
\end{abstract}

\section{Introduction}

Obesity is emerging as a significant public health concern in developed countries. Obesity contributes significantly to increasing medical costs by exacerbating other chronic diseases, such as cardiovascular disease, type 2 diabetes, and certain types of cancers. In the United States, more than onethird $(34.9 \%)$ of adults and $17 \%$ of the youth are obese [1]. A western diet comprised of a high fat content is a primary contributing factor for obesity which has led to extensive studies using models of high fat diet (HFD) to delve into the pathobiologies of obesity-related diseases.

Sphingolipids are bioactive lipids which are involved in cellular signaling and regulatory functions [2]. These lipid species have been implicated as potent mediators in the regulation of several diseases such as cancer and inflammatory diseases $[2,3]$. Based on these studies, interest for the role of sphingolipids in obesity-induced pathobiology is emerging. This is of particular interest as HFD significantly changes energy metabolism, including sphingolipid levels, suggesting the possibility that HFD-induced dysregulation of sphingolipid metabolism contributes to HFD and obesityrelated pathologies. This review will discuss sphingolipid metabolism altered by HFD and its impact on HFD-induced biology and pathobiology.

\section{Sphingolipid Metabolism}

Sphingolipid metabolism is a complex network composed of numerous metabolizing enzymes that function to generate sphingolipids through either de novo synthesis or the salvage pathway. Sphingolipid levels are tightly regulated by these enzymes, allowing them to function as bioactive mediators. De novo sphingolipid synthesis begins with the enzyme serine palmitoyltransferase (SPT). SPT functions as a heterodimer with subunits SPTLC1 and SPTLC2, or SPTLC3, primarily using palmitoyl-CoA as a substrate. SPTLC3 has recently been shown to utilize myristoyl-CoA as well [4]. Condensation of serine and palmitoyl-CoA (or myristoyl$\mathrm{CoA}$ ) which forms 3-ketosphinganine is then reduced to form dihydrosphingosine by NADH-dependent reductase. Ceramide synthases (CerS), of which there are six, catalyze 
the acylation of dihydrosphingosine to dihydroceramide. Subsequent desaturation yields the generation of ceramide from dihydroceramide. Ceramide can also be generated by sphingomyelinases (SMase) and glucosylceramidase (GCase) from various membrane glycolipids and sphingolipids in the salvage pathway. Once formed, ceramide acts as a central hub in the sphingolipid network. Ceramide can be phosphorylated by ceramide kinase to form ceramide 1-phosphate (C1P). Sphingomyelin synthase (SMS) and glucosyl- or galactosylceramide synthases (GCS) incorporate ceramide into sphingomyelin and glycosyl or galactosylceramide, respectively. Finally, one of five ceramidases (CDase) facilitates the deacylation of ceramide to produce sphingosine, followed by conversion to sphingosine-1 phosphate (S1P) by sphingosine kinases (SKs) or reacylation to ceramide by CerS. S1P can be degraded by one of two enzymes, sphingosine phosphate phosphatase (SPP) or S1P lyase. SPP dephosphorylates S1P to sphingosine, allowing for the reformation of ceramide, whereas S1P lyase irreversibly breaks down S1P to ethanolamine phosphate and hexadecanal, resulting in exit from sphingolipid metabolism.

\section{The Effects of HFD on Sphingolipid Metabolism}

3.1. Ceramide. Dysregulation of ceramide in response to nutrient oversupply, specifically saturated fatty acids, has been known to be a key factor in the impairment of cellular homeostasis and function [5]. During de novo synthesis, one of the six isoforms of CerS generates ceramide species with specific fatty acid chain lengths [6]. The long chain fatty acid palmitate (palmitoyl-CoA) is the main source of fatty acid in de novo sphingolipid synthesis. HFD administration and/or palmitate treatment have been shown to increase ceramide content independent of tissue or cell type (Table 1). Several tissues including liver, adipose, skeletal muscle, and heart demonstrate elevated total ceramide and long chain ceramide levels upon HFD administration. Diabetic models using HFD have also shown elevations in circulating ceramides [7]. Additionally, several studies have demonstrated C16:0 and C18:0 ceramides are consistently elevated by $\mathrm{HFD} /$ palmitate [8-13], compared to ceramides with the other chain lengths. Interestingly, very long chain ceramides, C24:0 and C24:1, have been shown to significantly increase upon HFD/palmitate treatment in some studies [8, 14-16] but are decreased in others $[9,11,17]$ (Table 1). Additionally, ceramide induction in response to HFD has also been demonstrated as an aging process with some studies indicating augmented accumulation of ceramide in muscle in aging individuals [1821]. The mechanism for this age-related increase in ceramide is still unclear, but the membrane protein CD36/FAT that facilitates the uptake of fatty acid has been suggested as a potential mechanism in HFD administration [20].

HFD-induced ceramide generation is primarily due to increased de novo synthesis, whereby excessive fatty acid supply supplies a continuous substrate supply for this pathway. Radioactive palmitate treatment in HepG2 cells (human hepatocarcinoma cell line) and $\mathrm{C} 2 \mathrm{C} 12$ cells (murine myotubes) increased incorporation of palmitate into ceramide, demonstrating that fatty acids from exogenous sources can be utilized for sphingolipid synthesis [14, 22]. In addition to increasing substrate, HFD/palmitate alters expression and activity of the enzymes involved in de novo synthesis. HFD increased transcription of SPT subunits, SPTLC1, SPTLC2, and SPTLC3 [9, 18, 23-25], as well as activity of SPT [26]. CerS have also been shown to be upregulated by $\operatorname{HFD}[9,17,24,25]$, specifically CerS1 and CerS6, which are key in the formation of long chain ceramide species. This upregulation resulted in the generation of $\mathrm{C16}: 0$ ceramide in response to $\operatorname{HFD}[6,17]$. In addition to CerS1 and CerS6, CerS2 and CerS4 expression has been reported to increase in response to $\mathrm{HFD} /$ palmitate $[23,24]$.

Upstream regulation of de novo sphingolipid synthesis by HFD has not been well studied but there are suggestions in the literature. TLR4, suggested as a receptor for saturated fatty acids [27], has also been shown to mediate several HFD-induced cell signaling events including HFDinduced de novo sphingolipid synthesis [28]. TLR4 deletion in skeletal muscle inhibited increases in ceramide levels upon HFD administration. Additionally, silencing the downstream signaling mechanism NF- $\kappa \mathrm{B}$ suppressed induction of mRNA expression in de novo sphingolipid enzymes, such as SPTLC1, SPTLC2, CerS1, CerS2, CerS5, and CerS6. Cannabinoid-1 receptor $\left(\mathrm{CB}_{1} \mathrm{R}\right)$ is another potential regulatory mechanism for de novo sphingolipid synthesis, as hepatic $\mathrm{CB}_{1} \mathrm{R}$ deletion or blockade suppressed HFD-induced SPTLC3, CerS1, and CerS6 upregulation and subsequent ceramide increases [9]. Additionally, the energy sensing molecule, AMP-activated protein (AMPK), has been shown to regulate de novo sphingolipid synthesis. The AMPK activator, AICAR, suppressed HFD-induced transcription of SPTLC2 in skeletal muscle, suggesting AMPK as an upstream regulator of SPTLC2 [23]. Adipocyte-derived plasminogen activator inhibitor-1 (PAI1) is important in the regulation of ceramide synthesis in adipose tissue as well [16]. PAI-1 deficient mice exhibited decreased expression of SPTLC2, SPTLC3, and CerS1 upon HFD feeding, resulting in reduced adipose ceramide level [16].

Enzymes of the salvage pathway have also been implicated in dietary manipulations of ceramide levels. Radio-labeled palmitate has been used to demonstrate the involvement of CerS and not de novo sphingolipid synthesis, as labeled palmitate was found in the acyl chain of ceramide, and not in the sphingoid backbone [14]. In addition to CerS, HFD administration enhanced mRNA expression and activity of acid SMase (aSMase) and neutral SMase (nSMase) in liver $[24,29]$ and adipose tissue [16]. Pharmacological inhibition of aSMase, using amitriptyline, inhibited ceramide induction by HFD in plasma and adipose tissue [30]. However, compared to studies examining de novo sphingolipid synthesis, regulation of the salvage pathway has not been well-studied.

Taken altogether, the above studies demonstrate that HFD and palmitate regulate global sphingolipid metabolism through both de novo sphingolipid synthesis and the salvage pathway. These diet-induced alterations in ceramide demonstrate that nutrition has the ability to alter sphingolipid metabolism and in turn downstream signaling pathways to 


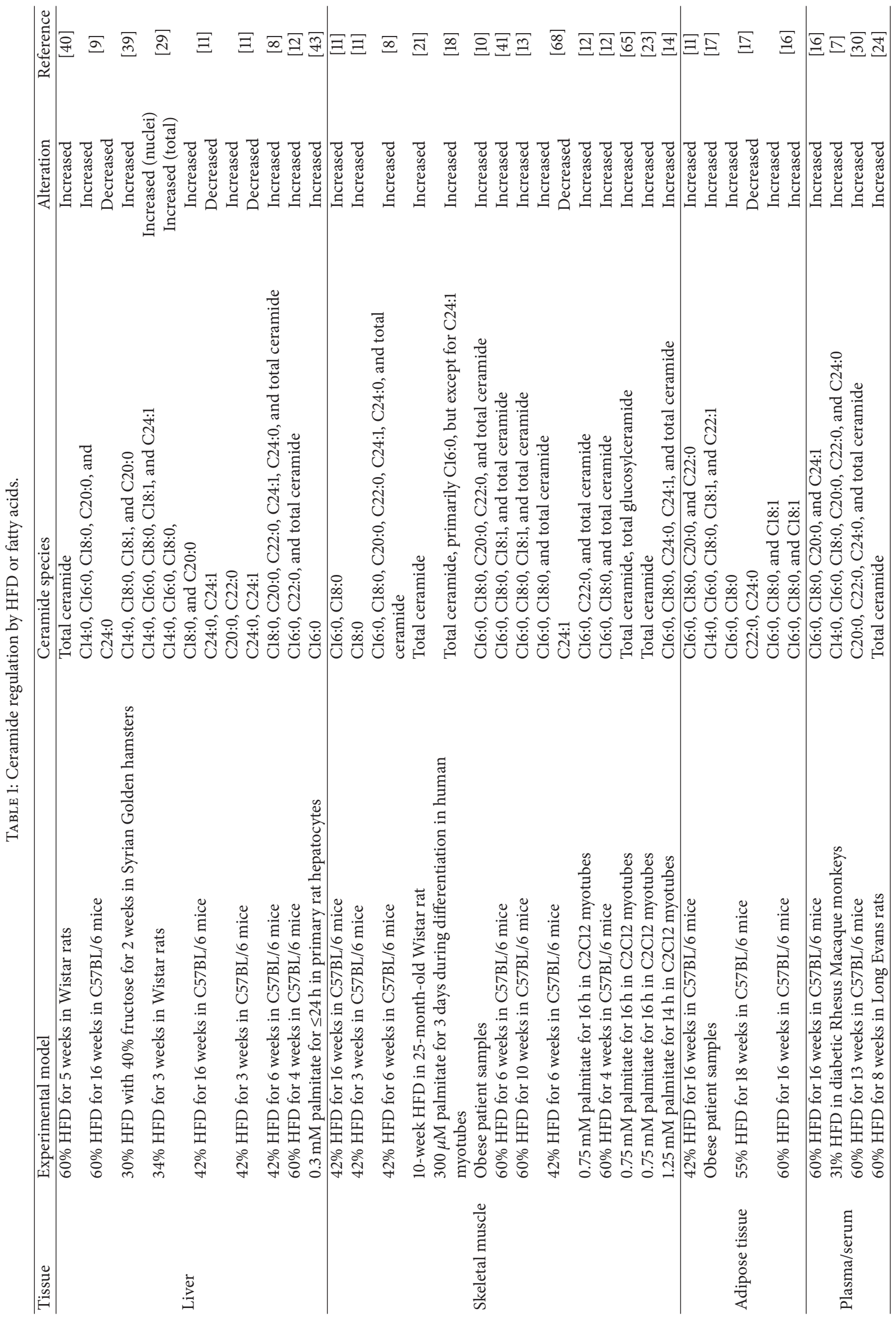




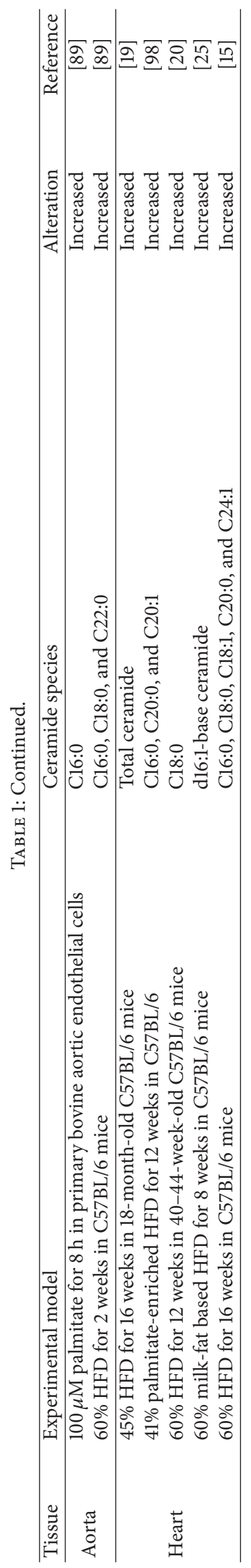


TABLE 2: Sphingomyelin regulation by HFD or fatty acids.

\begin{tabular}{|c|c|c|c|c|}
\hline Tissue & Experimental model & Sphingomyelin species & Alteration & Reference \\
\hline \multirow{4}{*}{ Liver } & $60 \%$ HFD for 5 weeks in Wistar rats & NS & Increased & {$[40]$} \\
\hline & \multirow{2}{*}{$34 \%$ HFD for 3 weeks in Wistar rats } & SM16:0, SM18:0, and SM18:1 & Increased (nuclei) & \multirow{2}{*}[29]{} \\
\hline & & SM16:0, SM18:0 & Increased (total) & \\
\hline & $58 \%$ HFD for 16 weeks in C57BL/6 mice & NS & Increased & {$[54]$} \\
\hline \multirow{2}{*}{ Skeletal muscle } & \multirow{2}{*}{$60 \% \mathrm{HFD}$ for 6 weeks in C57BL/6 mice } & SM18:1 & Increased & \multirow{2}{*}[41]{} \\
\hline & & SM18:0, total SM & Decreased & \\
\hline Adipose tissue & $42 \% \mathrm{HFD}$ for 16 weeks in C57BL/ 6 mice & $\begin{array}{l}\text { SM14:0, SM16:0, SM16:1 SM18:0, and } \\
\text { SM18:1 }\end{array}$ & Increased & [11] \\
\hline \multirow[t]{2}{*}{ Plasma/serum } & $45 \%$ HFD for 14 weeks in C57BL/6 mice & $\begin{array}{l}\text { SM16:0, SM18:0, SM16:1, SM18:1, and } \\
\text { SM22:1 }\end{array}$ & Increased & {$[33]$} \\
\hline & $58 \%$ HFD for 16 weeks in C57BL/ 6 mice & NS & Increased & {$[54]$} \\
\hline \multirow{2}{*}{ Heart } & $60 \%$ HFD for 16 weeks in C57BL/6 mice & SM 16:0, SM18:0, and SM18:1 & Increased & [15] \\
\hline & $\begin{array}{l}41 \% \text { palmitate-enriched HFD for } 12 \\
\text { weeks in C57BL/ } 6\end{array}$ & SM16:0, SM20:0, SM24:0, and SM24:1 & Increased & [98] \\
\hline
\end{tabular}

NS = not specified

induce obesity-related conditions, such as insulin resistance and ectopic lipid accumulation.

3.2. Sphingomyelin. Sphingomyelin, one of the most abundant sphingolipid species, plays key roles in membranes and has been suggested to function in lipid rafts [2, 31]. Concentrations of sphingomyelin are higher than ceramide, suggesting that sphingomyelin may act as a pool for the rapid generation of ceramide. It has been demonstrated that high concentrations of serum sphingomyelin correlate with coronary artery disease in obese individuals [32]; and several studies demonstrated that HFD or palmitate administration increased sphingomyelin levels in cells and tissues, including serum (Table 2) (although the magnitude of sphingomyelin increase was smaller than that of ceramide). Similar to alterations in ceramide species, long chain sphingomyelin species, mostly $\mathrm{C} 16: 0$ and $\mathrm{C} 18: 0$, have been reported to increase by HFD or palmitate treatment in liver, adipose tissue, and plasma [11, 29, 33] (Table 2). Macrophages stimulated with palmitate demonstrated increases in specific species of sphingomyelin, specifically 18:0, 20:0, 22:0, and 22:1 [34]. HFD-induced changes in sphingomyelin have been attributed to SMS2 activity and expression. Mice overexpressing liver-specific SMS2 exhibited elevated HFD-induced sphingomyelin in plasma. Additionally, this study demonstrated loss of SMS2 in vivo repressed HFD-induced increases in sphingomyelin [35].

The role for sphingomyelin in HFD-induced biologies has been less studied than ceramide; however, sphingomyelin levels are elevated in diverse tissues, including liver, skeletal muscle, adipose, and cardiac, as well as plasma. These studies suggest that sphingomyelin may directly regulate cell functions and/or serve as a reservoir for the generation of ceramide.
3.3. Sphingosine/S1P. Sphingosine, the breakdown product of ceramide, is known to regulate apoptosis and cell-cycle arrest [36-38]. There are few studies examining the role of sphingosine in HFD-mediated processes. It is possible that sphingosine is rapidly converted to S1P (or reacylated to ceramide) in HFD-mediated sphingolipid metabolism. However, the exact role of sphingosine in HFD-mediated effects has yet to be determined. S1P, a potent bioactive sphingolipid, is present in low nanomolar concentrations in the cell and has high affinity to S1PRs functioning as a signaling molecule. Despite its potent capacity as a bioactive lipid, studies for S1P (and its precursor, sphingosine) upon HFD administration are limited (compared to those examining ceramide). However, S1P (and sphingosine) levels exhibited significant increases in liver, skeletal muscle, and plasma in response to several experimental models of HFD. Conversely, there are also a few opposing studies that have demonstrated decreases in the levels of these lipids (Table 3). While S1P in liver and skeletal muscle was unchanged by HFD or palmitate in some studies [39-41], S1P in plasma was consistently elevated, which suggests that HFD-enhanced S1P levels in circulation may mediate some of the systemic effects associated with HFD and obesity. Furthermore, elevated plasma S1P positively correlated with body fat percentage, body mass index, waist circumference, and fasting plasma insulin in obese humans [42]. In cell culture palmitate stimulation of mouse derived pancreatic beta cells, MIN6, and primary rat hepatocytes resulted in increased S1P secretion $[43,44]$.

HFD-induced S1P seems to be due to augmented expression and activity of SK. Two isoforms for mammalian SK have been identified, SK1 and SK2. These two isoforms have suggested differences in subcellular localization, cytosol for SK1 and nucleus for SK2, as well as different physiological functions [45-47]. With respect to HFD, both enzymes have been shown to mediate HFD-induced S1P; however, 
TABLE 3: Sphingosine and S1P regulation by HFD or fatty acids.

\begin{tabular}{|c|c|c|c|c|}
\hline Tissue & Experimental model & Species & Alteration & Reference \\
\hline \multirow{5}{*}{ Liver } & $0.3 \mathrm{mM}$ palmitate for $\leq 24 \mathrm{~h}$ in primary rat hepatocyte & S1P & Increased & [43] \\
\hline & $30 \%$ HFD with $40 \%$ fructose for 2 weeks in Syrian Golden hamsters & Sphingosine & Increased & \multirow[t]{2}{*}{ [39] } \\
\hline & 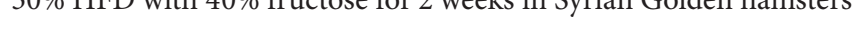 & $\mathrm{S} 1 \mathrm{P}$ & No Change & \\
\hline & $60 \%$ HFD for 5 weeks in Wistar rats & Sphingosine, S1P & No Change & {$[40]$} \\
\hline & $58 \%$ HFD for 16 weeks in C57BL/6 mice & Sphingosine & Increased & {$[54]$} \\
\hline \multirow{5}{*}{ Skeletal Muscle } & 60\% HFD for 6 weeks in C57BL/ 6 mice & Sphingosine, S1P & No Change & [41] \\
\hline & $0.75 \mathrm{mM}$ palmitate for $16 \mathrm{~h}$ in $\mathrm{C} 2 \mathrm{C} 12$ cells & S1P & Increased & {$[65]$} \\
\hline & & S1P & Increased & \multirow{2}{*}[14]{} \\
\hline & $1.25 \mathrm{mM}$ palmitate for $14 \mathrm{~h}$ in $\mathrm{C} 2 \mathrm{C} 12$ cells & Sphingosine & No Change & \\
\hline & $0.75 \mathrm{mM}$ palmitate for $16 \mathrm{~h}$ in primary mouse myotube & S1P & Increased & [48] \\
\hline Adipose tissue & $58 \%$ HFD for 16 weeks in C57BL/6 mice & Sphingosine & Increased & [54] \\
\hline \multirow{4}{*}{ Plasma } & $42 \%$ HFD for 6 weeks in C57BL/ 6 mice & S1P & Increased & {$[42]$} \\
\hline & $42 \%$ HFD for 6 weeks in C57BL/6 mice & S1P & Increased & {$[8]$} \\
\hline & & $\mathrm{S} 1 \mathrm{P}$ & Increased & \multirow{2}{*}[54]{} \\
\hline & $58 \%$ HFD for 16 weeks in C57BL/6 mice & Sphingosine & Increased & \\
\hline
\end{tabular}

regulation of these enzymes is suggested to be different. Skeletal muscle from mice fed a HFD for 16 weeks exhibited increases in only SK1 mRNA expression, and not SK2 [48]. In contrast, liver from rats fed a HFD for two weeks exhibited increased expression and activity of SK2, rather than SK1 [49].

The studies examining the effects of HFD on sphingosine and S1P levels suggest that S1P levels are elevated in several tissues. Moreover, S1P levels in plasma were elevated in response to $\mathrm{HFD}$ and have been suggested to be secreted from tissues including the liver and pancreas. This S1P has been shown to be generated by both isoforms of SK which are altered by HFD; however, they seem to be independently regulated each by independent mechanisms that have yet to be fully elucidated.

\section{Sphingolipids in HFD-Induced Pathobiology}

Obesity is well-known to induce several pathologic conditions, including insulin resistance. Insulin is responsible for clearance of redundant nutrients in circulation by facilitating their uptake and storage in liver, skeletal muscle, and adipose tissue. Under normal physiologic conditions, increased blood glucose from the diet stimulates insulin secretion and subsequent reduction of blood glucose, working as a negative feedback mechanism. However, when insulin fails to clear glucose in bloodstream this results in "insulin resistance." At the molecular level, insulin triggers signaling through the insulin receptor and subsequent phosphorylation of the insulin receptor substrate (IRS) [50]. Phosphorylated IRS recruits phosphatidylinositol 3-kinase (PI3K), which results in phosphorylation of Akt and its various substrates, including glycogen synthase kinase $3 \beta$ (GSK $3 \beta$ ) regulating glucose metabolism and glycogen, lipid, and protein synthesis [50, 51]. In insulin resistance, this signaling pathway is disrupted and ceramide has been reported to play a key role in the processes involved in insulin resistance [5].

Disruption of various enzymes in sphingolipid metabolism including SPT, CerS6, aSMase, and SMS2 suppressed weight gain by HFD $[17,30,52,53]$. Specifically, myriocin, an inhibitor of de novo sphingolipid synthesis, enhanced energy expenditure and improved leptin resistance associated with HFD inhibiting obesity [52]. Additionally, for decades the role for sphingolipids in postobesity events such as ectopic lipid accumulation and insulin resistance has been established. Induction of ceramide by palmitate has been shown to disturb insulin signaling via inhibition of Akt signaling mediated by TLR4-NF- $\kappa$ B in myotubes [28]. Many studies demonstrated that inhibition of de novo synthesis of sphingolipids suppressed blood glucose and insulin induction $[17,40,52,54]$. In addition to these systemic effects, aberrant sphingolipid regulation plays a significant role in HFDinduced dysregulation of various cellular signaling pathways. Understanding the specific effects in specific tissues will begin to lend insight into sphingolipid metabolism as a therapeutic target for HFD-induced pathologic conditions.

4.1. Liver. The liver is the central organ for the regulation of metabolism by action of insulin. Obesity-induced hepatic insulin resistance results in expansion of peripheral insulin resistance due to elevated fasting blood glucose and subsequent blood insulin levels. Sphingolipids play a significant role in energy metabolism; therefore, there has been an effort to elucidate the role for sphingolipids, particularly, ceramide and S1P, in hepatic insulin resistance.

Increased hepatic ceramide has been suggested to be a major mechanism in the regulation of insulin resistance, and suppression of ceramide induction has been demonstrated to improve insulin signaling. Direct treatment of Hu7 hepatoma cells with short chain C6:0 ceramide decreased 
phosphorylation of Akt and GSK3 $\beta$ [24]. The $\mathrm{CB}_{1} \mathrm{R}$ agonist anandamide increased endogenous C16:0 ceramide in hepatocytes via de novo synthesis, resulting in inhibition of insulin receptor substrate 1 (IRS1) phosphorylation, increased Akt phosphatase Phlppl expression, and suppression of Akt signaling in response to insulin [9]. Moreover, inhibition of ceramide synthesis through pharmacological inhibitors and gene deletion improved insulin signaling. Myriocin treatment and CerS6 deletion restored insulin-induced Akt signaling previously disrupted by ceramide $[9,17]$. In contrast, CerS2 haploinsufficiency reduced very long chain ceramide and inhibited insulin sensitivity. This contradictory result was suggested to be due to CerS6 upregulation and subsequent C16:0 ceramide synthesis upon HFD [55]. These studies suggest that the roles for ceramide in energy metabolism regulation may be species-dependent and that many enzymes may generate ceramide in the liver during insulin resistance.

Hepatic S1P and its receptors are also suggested to function as key molecules in the regulation of insulin signaling. Palmitate-induced production and secretion of S1P from hepatocytes led to not only abrogation of insulin-induced Akt activation, but also reduced glucokinase expression and consequent glycogen synthesis [43]. These signaling pathways are reversed by SKII and JTE-013 treatment, an SK1 inhibitor, and an S1P receptor 2 (S1PR2) antagonist, respectively [43]. SK2 overexpression enhanced insulin sensitivity in liver from HFD-fed mice [56], implicating localization of S1P may be important for regulation of hepatic insulin resistance.

In addition to ceramide and S1P, sphingomyelin also may be involved in the regulation of insulin sensitivity. SPTLC2 or SMS2 deletion and subsequent decreases in sphingomyelin levels in the plasma membrane restored insulin signaling disrupted by HFD. Also exogenous sphingomyelin treatment impaired insulin-stimulated Akt phosphorylation [54].

Nonalcoholic steatohepatitis (NASH) is a major obesityrelated disease that involves elevated proinflammatory cytokine activation, oxidative stress with mitochondrial dysfunction, leading to fibrogenesis, and finally liver cirrhosis [5759]. The progress of these diseases is closely associated with dysregulated intracellular lipid accumulation and metabolism [57], and recently the involvement of sphingolipids in this process has been demonstrated. Inhibition of de novo sphingolipid synthesis with myriocin reduced triglyceride (TG) accumulation in liver [40]. Similarly, CerS6 deletion reduced CD36/FAT expression and increased palmitate $\beta$ oxidation, resulting in suppressed lipid accumulation [17]. Increased ceramide breakdown by aCDase induction prevented TG accumulation in liver from HFD feeding [60]. It has also been well documented that obesity induces ER stress [61] and important mechanism in the activation of lipogenesis [62]. Indeed, inhibition of the salvage pathway via aSMase deletion ameliorated HFD-induced hepatic steatosis via protection from ER stress [63]. However, acute ER stress augmented SK2 expression, inhibiting HFD-induced lipid accumulation in the liver [56], potentially functioning as an initial defense mechanism against HFD-induced lipotoxicity. In addition to the study demonstrating overexpression of SK2 suppressed lipid accumulation [56], deletion of SK2 significantly intensified HFD-induced hepatic steatosis [49].
The latter study demonstrated nuclear S1P was critical for histone acetylation and followed global gene transcription in lipid metabolism [49]. These studies highlight the importance of subcellular localization of sphingolipid metabolizing enzymes and their lipid products suggesting that specific pools of sphingolipids function to differentially regulate hepatic insulin resistance.

These studies demonstrate that HFD-induced alteration in sphingolipid metabolism disrupts normal physiology in liver. Specifically, increases in ceramides have been demonstrated to play a key role in insulin resistance, while future studies will be needed to determine the specific roles of sphingomyelin, sphingosine, and S1P.

4.2. Skeletal Muscle. Skeletal muscle is a primary site for glucose utilization and is significantly affected by HFD. Similar to the liver, HFD induces insulin resistance in skeletal muscle, inhibiting glucose uptake [64]. In myotubes palmitateinduced ceramide generation and exogenous C2-ceramide treatment impaired insulin-stimulated Akt activation [65]. Additionally, ceramide-induced $\mathrm{PKC} \zeta$ activation triggered the inhibitory phosphorylation of Akt [66]. Inhibition of nSMase, which should decrease ceramide generation, abolished palmitate-induced JNK and NF- $\kappa$ B activation and ER stress, conferring improvement in insulin resistance [67]. Furthermore, ceramide has also been implicated in the regulation of ectopic lipid accumulation in skeletal muscle and aggravation of insulin resistance [21, 68].

Recently, skeletal muscle has emerged as secretory tissue for cytokines, termed myokines [69]. Interleukin-6 (IL-6), the first described myokine, was found to be released from contracting skeletal muscle [70]. In relation to HFD, in C2C12 and mouse primary myotubes, palmitate stimulated IL-6 expression and secretion in an SK1- and S1PR3-dependent manner [48]. These data suggest that SK1, S1P, and S1PRs may play a role in skeletal muscle myokine generation. However, the definitive role for IL-6 from skeletal muscle is controversial in HFD-induced metabolic disorders [64].

4.3. Adipose Tissue. The traditional perception of adipose tissue is that of long-term energy storage. However, since the discovery of increased of tumor necrosis factor alpha $(\mathrm{TNF} \alpha)$ in adipose tissue from obese individuals [71], this tissue has become the focus of numerous studies on the secretion of proinflammatory cytokines and consequent pathobiologies including insulin resistance [72, 73]. After identification of $\mathrm{TNF} \alpha$, various cytokines including leptin, PAI-1, and adiponectin have been defined as adipokines [7476]. Adipose tissue consists primarily of adipocytes with minor population of preadipocytes and immune cells, such as lymphocytes and macrophages. Infiltration of macrophages occurs via recruitment by chemokines and cytokines secreted by adipose tissue resulting in exacerbation of obesity-induced proinflammatory responses [73]. HFD-induced ceramide levels have also been implicated in adipokine induction and secretion. CerS6 deletion, and subsequent decreased ceramide levels, inhibited expression of proinflammatory cytokines including IL-6 in response to HFD [17]. In addition, de novo synthesis of ceramide contributed to HFD 
stimulation of monocyte chemoattractant protein-1 (MCP-1), suggesting that ceramide may play a role in recruitment of immune cells in adipose tissue [52]. Furthermore, this may suggest a role for adipose-derived ceramide in chronic and systemic inflammation via adipokine regulation in response to HFD.

In addition to adipokine regulation, ceramide has been shown to function in the regulation of adipose tissue. Inhibition of dihydroceramide desaturase reduced ceramide formation and increased dihydroceramide accumulation in 3T3-L1 mouse derived adipocytes and white adipose tissues in mice, leading to impaired differentiation and lipid accumulation [77]. This study implicates ceramide and dihydroceramide regulation of adipose tissue homeostasis.

C1P, generated by ceramide kinase, has not been well studied in response to HFD. The study that has been performed demonstrated that deletion of ceramide kinase abrogated increased mRNA expression of adipokines IL-6, TNF $\alpha$, and MCP-1 and decreased macrophage recruitment to adipose tissue, in response to HFD [78]. This study suggests that ceramide kinase, and perhaps C1P, may function similarly to ceramide in the generation of adipokines.

S1PR modulators are being extensively utilized to define S1PR-mediated signaling events and pathobiologies [2]. Upon phosphorylation by SK2, FTY720 is able to bind S1PR1 inducing internalization and degradation of the receptor and sequestration of lymphocytes in secondary lymphoid tissues $[79,80]$. In response to HFD, FTY720 prevented HFD-induced lymphocyte and macrophage infiltration in adipose tissue (although the mechanism for macrophages is unclear), resulting in reduced local inflammation and improved insulin resistance [81]. In addition to immune cell regulation, FTY720 has been shown to directly mediate HFD-induced effects on adipocytes. Phospho-FTY720 inhibited preadipocyte differentiation into mature adipocytes and stimulated lipolysis, reducing fat mass in HFD-fed mice [82]. In addition to receptor mediated effects on adipocytes, S1P itself has been implicated downstream of SK1 in lipid accumulation. Pharmacological or genetic inhibition of SK1 attenuated lipid accumulation in differentiating 3T3L1 adipocytes [83]. These studies suggest involvement of S1PS1PR signaling in HFD-induced adipose tissue dysregulation.

Sphingolipids in adipose tissue are potent mediators for adipokine expression and secretion in response to HFD. Moreover, sphingolipid enzymes and their product lipids may also regulate subsequent immune cell infiltration and proinflammatory signaling in adipose tissues in HFD and obesity.

4.4. Cardiovascular System. Systemic insulin resistance and excessive cytokine release due to obesity significantly alter the cardiovascular environment including blood pressure, coagulation, and fibrinolysis, ultimately leading to endothelial dysfunction and atherosclerosis [84]. Endothelial dysfunction is the failed balance between endothelium-dependent vasodilation and contraction. In addition to this imbalance, endothelial activation confers susceptibility to atherosclerosis [85]. Sphingolipids are significantly altered by HFD and palmitate treatment in the cardiovascular system, and these altered sphingolipids, specifically ceramide, seem to be essential in the regulation of obesity-induced cardiovascular diseases [86-89].

Endothelial dysfunction is mainly caused by dysregulation of endothelial nitric oxide synthase (eNOS), the enzyme that generates the potent vasodilator nitric oxide (NO) [85]. Palmitate- and HFD-induced generation of ceramide resulted in endothelial dysfunction, abolishing vascular endothelial growth factor (VEGF) or insulin-stimulated eNOS activation and subsequent NO production from endothelial cells [88, 89]. Inhibition of de novo synthesis with myriocin and SPTLC2 haploinsufficiency restored HFD-induced eNOS phosphorylation and achieved the normal range of vasodilation [88]. In addition, palmitate impaired angiogenesis in human umbilical vein endothelial cells (HUVECs) upon VEGF treatment, resulting in reduced tube formation in matrigel. This reduction in angiogenesis was rescued by myriocin, demonstrating the involvement of de novo generated sphingolipids in VEGF function [89].

Plasma sphingomyelin levels are reported to correlate with the increased risk of coronary heart disease [32]. In addition, high levels of sphingomyelin in low density lipoprotein (LDL) contributed to aggregation and retention of LDL in arterial wall. High levels of LDL-sphingomyelin supplied sufficient substrate for arterial SMase to increase LDL-ceramide, conferring aggravated atherosclerotic damage [90]. Consistently, LDL from SMS2 transgenic mice exhibit proatherogenic properties with increased in vitro aggregation upon SMase treatment [35]. Also, SMS2 overexpression in mice with adenovirus exaggerated atherosclerotic inflammation with elevations in cyclooxygenase-2 (COX-2) and matrix metalloproteinase-2 (MMP-2) in aorta [91]. Myriocin treatment decreased sphingomyelin and ceramide levels in plasma and consequently resulted in less atherosclerotic lesions in aorta from HFD-fed Apo-E deficient mice [87, 92, 93]. Moreover, bone marrow-derived SPTLC2 haploinsufficient macrophages also reduced atherosclerotic lesions in mice suggesting the importance of immune cell-derived sphingolipids in this disease (similar to adipose tissue) [86]. Meanwhile, the effect of glycosphingolipids and their metabolizing enzymes in atherosclerosis has been controversial [94-97]. The studies that have been conducted suggest that glycosphingolipids are necessary in atherosclerosis progress, but not a sufficient therapeutic target for this disease.

Altered sphingolipid metabolism has also been implicated in cardiomyopathy. A single study suggested that palmitate-induced ceramide and sphingomyelin accumulation in cardiac tissue resulted in $\mathrm{Ca}^{++}$dysregulation and consequent systolic contractile dysfunction [98]. In a recent study by Russo et al., cardiac tissue was found to be enriched in SPTLC3, which has been shown to utilize the C14:0 saturated fatty acid myristate. This results in the de novo generation of d16:0/d16:1-base sphingolipids. Elevation of these d16:0/d16:1 sphingolipids resulted in increased apoptosis [25]. This study suggested the involvement of novel sphingolipid species in obesity-induced cardiomyopathy.

HFD-mediated dysregulation of sphingolipids contributes to obesity-related cardiovascular disease. In addition, ceramide and sphingomyelin contribute to endothelial dysfunction, 
atherosclerosis, and cardiomyopathy in cardiovascular tissues. Together these studies suggest that dietary fat overload and obesity alter cardiovascular function are at least partially due to altered sphingolipid metabolism.

\section{Conclusion}

Sphingolipid metabolism is significantly affected by dietary nutrient oversupply, including HFD. Due to the complexity of the sphingolipid metabolism network, it is hard to define the regulation and role of a single sphingolipid species in HFD-involved biologies and pathobiologies. However, HFD significantly alters numerous sphingolipids species, impacting the downstream sphingolipid-mediated cellular signaling pathways. These changes in cellular signaling often contribute to HFD-induced toxicity in affected tissues: liver, muscle, adipose, and cardiovascular. Therefore, dissecting HFD-mediated sphingolipid metabolism and understanding the mechanisms by which HFD regulates these changes may lend insight into potential therapeutic and nutritional targets in obesity-related diseases.

\section{Conflict of Interests}

The authors declare that there is no conflict of interests regarding the publication of this paper.

\section{Acknowledgment}

This work was supported by a Veterans Affairs Career Development Award (Ashley J. Snider).

\section{References}

[1] C. L. Ogden, M. D. Carroll, B. K. Kit, and K. M. Flegal, "Prevalence of childhood and adult obesity in the United States, 20112012," The Journal of the American Medical Association, vol. 311, no. 8, pp. 806-814, 2014.

[2] Y. A. Hannun and L. M. Obeid, "Principles of bioactive lipid signalling: lessons from sphingolipids," Nature Reviews Molecular Cell Biology, vol. 9, no. 2, pp. 139-150, 2008.

[3] A. J. Snider, K. A. Orr Gandy, and L. M. Obeid, "Sphingosine kinase: role in regulation of bioactive sphingolipid mediators in inflammation," Biochimie, vol. 92, no. 6, pp. 707-715, 2010.

[4] T. Hornemann, A. Penno, M. F. Rütti et al., "The SPTLC3 subunit of serine palmitoyltransferase generates short chain sphingoid bases," Journal of Biological Chemistry, vol. 284, no. 39, pp. 26322-26330, 2009.

[5] S. A. Summers, "Ceramides in insulin resistance and lipotoxicity," Progress in Lipid Research, vol. 45, no. 1, pp. 42-72, 2006.

[6] M. Levy and A. H. Futerman, "Mammalian ceramide synthases," IUBMB Life, vol. 62, no. 5, pp. 347-356, 2010.

[7] J. T. Brozinick, E. Hawkins, H. Hoang Bui et al., "Plasma sphingolipids are biomarkers of metabolic syndrome in non-human primates maintained on a Western-style diet," International Journal of Obesity, vol. 37, no. 8, pp. 1064-1070, 2013.

[8] C. R. Bruce, S. Risis, J. R. Babb et al., "Overexpression of sphingosine kinase 1 prevents ceramide accumulation and ameliorates muscle insulin resistance in high-fat diet-fed mice," Diabetes, vol. 61, no. 12, pp. 3148-3155, 2012.
[9] R. Cinar, G. Godlewski, J. Liu et al., "Hepatic cannabinoid-1 receptors mediate diet-induced insulin resistance by increasing de novo synthesis of long-chain ceramides," Hepatology, vol. 59, no. 1, pp. 143-153, 2014.

[10] A. B. Thrush, D. N. Brindley, A. Chabowski, G. J. Heigenhauser, and D. J. Dyck, "Skeletal muscle lipogenic protein expression is not different between lean and obese individuals: a potential factor in ceramide accumulation," Journal of Clinical Endocrinology and Metabolism, vol. 94, no. 12, pp. 5053-5061, 2009.

[11] N. Turner, G. M. Kowalski, S. J. Leslie et al., "Distinct patterns of tissue-specific lipid accumulation during the induction of insulin resistance in mice by high-fat feeding," Diabetologia, vol. 56, no. 7, pp. 1638-1648, 2013.

[12] B. T. Bikman, Y. Guan, G. Shui et al., "Fenretinide prevents lipidinduced insulin resistance by blocking ceramide biosynthesis," The Journal of Biological Chemistry, vol. 287, no. 21, pp. 1742617437, 2012.

[13] I. R. Lanza, A. Blachnio-Zabielska, M. L. Johnson et al., "Influence of fish oil on skeletal muscle mitochondrial energetics and lipid metabolites during high-fat diet," American Journal of Physiology -Endocrinology and Metabolism, vol. 304, no. 12, pp. E1391-E1403, 2013.

[14] W. Hu, J. Bielawski, F. Samad, A. H. Merrill Jr., and L. A. Cowart, "Palmitate increases sphingosine-1-phosphate in $\mathrm{C} 2 \mathrm{C} 12$ myotubes via upregulation of sphingosine kinase message and activity," Journal of Lipid Research, vol. 50, no. 9, pp. 1852-1862, 2009.

[15] M. Park, D. Wu, T. Park et al., "APPL1 transgenic mice are protected from high-fat diet-induced cardiac dysfunction," American Journal of Physiology - Endocrinology and Metabolism, vol. 305, no. 7, pp. E795-E804, 2013.

[16] C. Shah, G. Yang, I. Lee, J. Bielawski, Y. A. Hannun, and F. Samad, "Protection from high fat diet-induced increase in ceramide in mice lacking plasminogen activator inhibitor 1," The Journal of Biological Chemistry, vol. 283, no. 20, pp. 13538-13548, 2008.

[17] S. M. Turpin, H. T. Nicholls, D. M. Willmes et al., "Obesityinduced CerS6-dependent $\mathrm{C}_{16: 0}$ ceramide production promotes weight gain and glucose intolerance," Cell Metabolism, vol. 20, no. 4, pp. 678-686, 2014.

[18] J. Abildgaard, D. C. Henstridge, A. T. Pedersen et al., "In vitro palmitate treatment of myotubes from postmenopausal women leads to ceramide accumulation, inflammation and affected insulin signaling," PLoS ONE, vol. 9, no. 7, Article ID e101555, 2014.

[19] A.-C. Aurich, B. Niemann, R. Pan et al., "Age-dependent effects of high fat-diet on murine left ventricles: role of palmitate," Basic Research in Cardiology, vol. 108, no. 5, article 369, 2013.

[20] M. M. Y. Sung, D. P. Y. Koonen, C.-L. M. Soltys, R. L. Jacobs, M. Febbraio, and J. R. B. Dyck, "Increased CD36 expression in middle-aged mice contributes to obesity-related cardiac hypertrophy in the absence of cardiac dysfunction," Journal of Molecular Medicine, vol. 89, no. 5, pp. 459-469, 2011.

[21] N. Tardif, J. Salles, C. Guillet et al., "Muscle ectopic fat deposition contributes to anabolic resistance in obese sarcopenic old rats through eIF $2 \alpha$ activation," Aging Cell, vol. 13, no. 6, pp. 10011011, 2014.

[22] M. J. Watt, A. C. Barnett, C. R. Bruce, S. Schenk, J. F. Horowitz, and A. J. Hoy, "Regulation of plasma ceramide levels with fatty acid oversupply: evidence that the liver detects and secretes de novo synthesised ceramide," Diabetologia, vol. 55, no. 10, pp. 2741-2746, 2012. 
[23] K. A. Erickson, M. E. Smith, T. S. Anthonymuthu et al., "AICAR inhibits ceramide biosynthesis in skeletal muscle," Diabetology \& Metabolic Syndrome, vol. 4, no. 1, article 45, 2012.

[24] L. Longato, M. Tong, J. R. Wands, and S. M. de la Monte, "High fat diet induced hepatic steatosis and insulin resistance: role of dysregulated ceramide metabolism," Hepatology Research, vol. 42, no. 4, pp. 412-427, 2012.

[25] S. B. Russo, R. Tidhar, A. H. Futerman, and L. A. Cowart, "Myristate-derived d16:0 sphingolipids constitute a cardiac sphingolipid pool with distinct synthetic routes and functional properties," The Journal of Biological Chemistry, vol. 288, no. 19, pp. 13397-13409, 2013.

[26] A. Blachnio-Zabielska, M. Baranowski, P. Zabielski, and J. Gorski, "Effect of high fat diet enriched with unsaturated and diet rich in saturated fatty acids on sphingolipid metabolism in rat skeletal muscle," Journal of Cellular Physiology, vol. 225, no. 3, pp. 786-791, 2010.

[27] H. Shi, M. V. Kokoeva, K. Inouye, I. Tzameli, H. Yin, and J. S. Flier, "TLR4 links innate immunity and fatty acid-induced insulin resistance," The Journal of Clinical Investigation, vol. 116, no. 11, pp. 3015-3025, 2006.

[28] W. L. Holland, B. T. Bikman, L.-P. Wang et al., "Lipid-induced insulin resistance mediated by the proinflammatory receptor TLR4 requires saturated fatty acid-induced ceramide biosynthesis in mice," Journal of Clinical Investigation, vol. 121, no. 5, pp. 1858-1870, 2011.

[29] G. Chocian, A. Chabowski, M. Zendzian-Piotrowska, E. Harasim, B. Łukaszuk, and J. Górski, "High fat diet induces ceramide and sphingomyelin formation in rat's liver nuclei," Molecular and Cellular Biochemistry, vol. 340, no. 1-2, pp. 125-131, 2010.

[30] K. M. Boini, C. Zhang, M. Xia, J. L. Poklis, and P.-L. Li, "Role of sphingolipid mediator ceramide in obesity and renal injury in mice fed a high-fat diet," Journal of Pharmacology and Experimental Therapeutics, vol. 334, no. 3, pp. 839-846, 2010.

[31] D. A. Brown and E. London, "Structure and function of sphingolipid- and cholesterol-rich membrane rafts," The Journal of Biological Chemistry, vol. 275, no. 23, pp. 17221-17224, 2000.

[32] X.-C. Jiang, F. Paultre, T. A. Pearson et al., "Plasma sphingomyelin level as a risk factor for coronary artery disease," Arteriosclerosis, Thrombosis, and Vascular Biology, vol. 20, no. 12, pp. 2614-2618, 2000.

[33] K. Eisinger, G. Liebisch, G. Schmitz, C. Aslanidis, S. Krautbauer, and C. Buechler, "Lipidomic analysis of serum from high fat diet induced obese mice," International Journal of Molecular Sciences, vol. 15, no. 2, pp. 2991-3002, 2014.

[34] J. Jin, X. Zhang, Z. Lu et al., "Acid sphingomyelinase plays a key role in palmitic acid-amplified inflammatory signaling triggered by lipopolysaccharide at low concentrations in macrophages," American Journal of Physiology: Endocrinology and Metabolism, vol. 305, no. 7, pp. E853-E867, 2013.

[35] J. Liu, H. Zhang, Z. Li et al., "Sphingomyelin synthase 2 is one of the determinants for plasma and liver sphingomyelin levels in mice," Arteriosclerosis, Thrombosis, and Vascular Biology, vol. 29, no. 6, pp. 850-856, 2009.

[36] O. Cuvillier, L. Edsall, and S. Spiegel, "Involvement of sphingosine in mitochondria-dependent Fas-induced apoptosis of type II Jurkat T cells," The Journal of Biological Chemistry, vol. 275, no. 21, pp. 15691-15700, 2000.

[37] K. Kågedal, M. Zhao, I. Svensson, and U. T. Brunk, "Sphingosine-induced apoptosis is dependent on lysosomal proteases," Biochemical Journal, vol. 359, no. 2, pp. 335-343, 2001.
[38] H. Okuwa, T. Kanno, Y. Fujita et al., "Sphingosine suppresses mesothelioma cell proliferation by inhibiting PKC- $\delta$ and inducing cell cycle arrest at the $\mathrm{G}_{0} / \mathrm{G}_{1}$ phase," Cellular Physiology and Biochemistry, vol. 30, no. 4, pp. 995-1004, 2012.

[39] M. J. Dekker, C. Baker, M. Naples et al., "Inhibition of sphingolipid synthesis improves dyslipidemia in the diet-induced hamster model of insulin resistance: evidence for the role of sphingosine and sphinganine in hepatic VLDL-apoB100 overproduction," Atherosclerosis, vol. 228, no. 1, pp. 98-109, 2013.

[40] K. Kurek, D. M. Piotrowska, P. Wiesiołek-Kurek et al., "Inhibition of ceramide de novo synthesis reduces liver lipid accumulation in rats with nonalcoholic fatty liver disease," Liver International, vol. 34, no. 7, pp. 1074-1083, 2014.

[41] Y. Liu, S. Turdi, T. Park et al., "Adiponectin corrects high-fat diet-induced disturbances in muscle metabolomic profile and whole-body glucose homeostasis," Diabetes, vol. 62, no. 3, pp. 743-752, 2013.

[42] G. M. Kowalski, A. L. Carey, A. Selathurai, B. A. Kingwell, and C. R. Bruce, "Plasma sphingosine-1-phosphate is elevated in obesity," PLoS ONE, vol. 8, no. 9, Article ID e72449, 2013.

[43] S. Fayyaz, J. Henkel, L. Japtok et al., "Involvement of sphingosine 1-phosphate in palmitate-induced insulin resistance of hepatocytes via the $\mathrm{SIP}_{2}$ receptor subtype," Diabetologia, vol. 57 , no. 2 , pp. 373-382, 2014.

[44] L. Japtok, E. I. Schmitz, S. Fayyaz, S. Krämer, L. Hsu, and B. Kleuser, "Sphingosine 1-phosphate counteracts insulin signaling in pancreatic beta-cells via the sphingosine 1-phosphate receptor subtype 2," The FASEB Journal, vol. 29, no. 8, pp. 33573369, 2015.

[45] N. Igarashi, T. Okada, S. Hayashi, T. Fujita, S. Jahangeer, and S.-I. Nakamura, "Sphingosine kinase 2 is a nuclear protein and inhibits DNA synthesis," Journal of Biological Chemistry, vol. 278, no. 47, pp. 46832-46839, 2003.

[46] K. R. Johnson, K. P. Becker, M. M. Facchinetti, Y. A. Hannun, and L. M. Obeid, "PKC-dependent activation of sphingosine kinase 1 and translocation to the plasma membrane: extracellular release of sphingosine-1-phosphate induced by phorbol 12myristate 13-acetate (PMA)," The Journal of Biological Chemistry, vol. 277, no. 38, pp. 35257-35262, 2002.

[47] W.-Q. Lai, A. W. Irwan, H. H. Goh, A. J. Melendez, I. B. McInnes, and B. P. Leung, "Distinct roles of sphingosine kinase 1 and 2 in murine collagen-induced arthritis," The Journal of Immunology, vol. 183, no. 3, pp. 2097-2103, 2009.

[48] J. S. Ross, W. Hu, B. Rosen, A. J. Snider, L. M. Obeid, and L. A. Cowart, "Sphingosine kinase 1 is regulated by peroxisome proliferator-activated receptor $\alpha$ in response to free fatty acids and is essential for skeletal muscle interleukin- 6 production and signaling in diet-induced obesity," The Journal of Biological Chemistry, vol. 288, no. 31, pp. 22193-22206, 2013.

[49] M. Nagahashi, K. Takabe, R. Liu et al., "Conjugated bile acidactivated S1P receptor 2 is a key regulator of sphingosine kinase 2 and hepatic gene expression," Hepatology, vol. 61, no. 4, pp. 1216-1226, 2015.

[50] A. R. Saltiel and C. R. Kahn, "Insulin signalling and the regulation of glucose and lipid metabolism," Nature, vol. 414, no. 6865, pp. 799-806, 2001.

[51] E. L. Whiteman, H. Cho, and M. J. Birnbaum, "Role of Akt/protein kinase B in metabolism," Trends in Endocrinology and Metabolism, vol. 13, no. 10, pp. 444-451, 2002.

[52] G. Yang, L. Badeanlou, J. Bielawski, A. J. Roberts, Y. A. Hannun, and F. Samad, "Central role of ceramide biosynthesis in 
body weight regulation, energy metabolism, and the metabolic syndrome," American Journal of Physiology: Endocrinology and Metabolism, vol. 297, no. 1, pp. E211-E224, 2009.

[53] S. Mitsutake, K. Zama, H. Yokota et al., "Dynamic modification of sphingomyelin in lipid microdomains controls development of obesity, fatty liver, and type 2 diabetes," Journal of Biological Chemistry, vol. 286, no. 32, pp. 28544-28555, 2011.

[54] Z. Li, H. Zhang, J. Liu et al., "Reducing plasma membrane sphingomyelin increases insulin sensitivity," Molecular and Cellular Biology, vol. 31, no. 20, pp. 4205-4218, 2011.

[55] S. Raichur, S. T. Wang, P. W. Chan et al., "CerS2 haploinsufficiency inhibits $\beta$-oxidation and confers susceptibility to dietinduced steatohepatitis and insulin resistance," Cell Metabolism, vol. 20, no. 4, pp. 687-695, 2014.

[56] S. Y. Lee, I. Hong, B. Kim et al., "Activation of sphingosine kinase 2 by endoplasmic reticulum stress ameliorates hepatic steatosis and insulin resistance in mice," Hepatology, vol. 62, no. 1, pp. 135-146, 2015.

[57] P. Angulo, "Medical progress: nonalcoholic fatty liver disease," The New England Journal of Medicine, vol. 346, no. 16, pp. 12211231, 2002.

[58] K. Begriche, A. Igoudjil, D. Pessayre, and B. Fromenty, "Mitochondrial dysfunction in NASH: causes, consequences and possible means to prevent it," Mitochondrion, vol. 6, no. 1, pp. $1-28,2006$.

[59] J. D. Browning and J. D. Horton, "Molecular mediators of hepatic steatosis and liver injury," The Journal of Clinical Investigation, vol. 114, no. 2, pp. 147-152, 2004.

[60] J. Y. Xia, W. L. Holland, C. M. Kusminski et al., "Targeted induction of ceramide degradation leads to improved systemic metabolism and reduced hepatic steatosis," Cell Metabolism, vol. 22, no. 2, pp. 266-278, 2015.

[61] U. Özcan, Q. Cao, E. Yilmaz et al., "Endoplasmic reticulum stress links obesity, insulin action, and type 2 diabetes," Science, vol. 306, no. 5695, pp. 457-461, 2004.

[62] J.-S. Lee, R. Mendez, H. H. Heng, Z.-Q. Yang, and K. Zhang, "Pharmacological ER stress promotes hepatic lipogenesis and lipid droplet formation," American Journal of Translational Research, vol. 4, no. 1, pp. 102-113, 2012.

[63] R. Fucho, L. Martínez, A. Baulies et al., "ASMase regulates autophagy and lysosomal membrane permeabilization and its inhibition prevents early stage non-alcoholic steatohepatitis," Journal of Hepatology, vol. 61, no. 5, pp. 1126-1134, 2014.

[64] G. Kewalramani, P. J. Bilan, and A. Klip, "Muscle insulin resistance: assault by lipids, cytokines and local macrophages," Current Opinion in Clinical Nutrition and Metabolic Care, vol. 13, no. 4, pp. 382-390, 2010.

[65] J. A. Chavez, M. M. Siddique, S. T. Wang, J. Ching, J. A. Shayman, and S. A. Summers, "Ceramides and glucosylceramides are independent antagonists of insulin signaling," Journal of Biological Chemistry, vol. 289, no. 2, pp. 723-734, 2014.

[66] D. J. Powell, S. Turban, A. Gray, E. Hajduch, and H. S. Hundal, "Intracellular ceramide synthesis and protein kinase $\mathrm{C} \zeta$ activation play an essential role in palmitate-induced insulin resistance in rat L6 skeletal muscle cells," Biochemical Journal, vol. 382, part 2, pp. 619-629, 2004.

[67] M. K. Verma, A. N. Yateesh, K. Neelima et al., "Inhibition of neutral sphingomyelinases in skeletal muscle attenuates fattyacid induced defects in metabolism and stress," SpringerPlus, vol. 3, article 255, 2014.
[68] C. R. Bruce, S. Risis, J. R. Babb et al., "The sphingosine-1phosphate analog FTY720 reduces muscle ceramide content and improves glucose tolerance in high fat-fed male mice," Endocrinology, vol. 154, no. 1, pp. 65-76, 2013.

[69] B. K. Pedersen and M. A. Febbraio, "Muscle as an endocrine organ: focus on muscle-derived interleukin-6," Physiological Reviews, vol. 88, no. 4, pp. 1379-1406, 2008.

[70] A. Steensberg, G. Van Hall, T. Osada, M. Sacchetti, B. Saltin, and B. K. Pedersen, "Production of interleukin-6 in contracting human skeletal muscles can account for the exercise-induced increase in plasma interleukin-6," Journal of Physiology, vol. 529, no. 1, pp. 237-242, 2000.

[71] G. S. Hotamisligil, N. S. Shargill, and B. M. Spiegelman, "Adipose expression of tumor necrosis factor- $\alpha$ : direct role in obesity-linked insulin resistance," Science, vol. 259, no. 5091, pp. 87-91, 1993.

[72] A. H. Berg and P. E. Scherer, "Adipose tissue, inflammation, and cardiovascular disease," Circulation Research, vol. 96, no. 9, pp. 939-949, 2005.

[73] N. Ouchi, J. L. Parker, J. J. Lugus, and K. Walsh, "Adipokines in inflammation and metabolic disease," Nature Reviews Immunology, vol. 11, no. 2, pp. 85-97, 2011.

[74] Y. Zhang, R. Proenca, M. Maffei, M. Barone, L. Leopold, and J. M. Friedman, "Positional cloning of the mouse obese gene and its human homologue," Nature, vol. 372, no. 6505, pp. 425-432, 1994.

[75] I. Shimomura, T. Funahashi, M. Takahashi et al., "Enhanced expression of PAI-1 in visceral fat: possible contributor to vascular disease in obesity," Nature Medicine, vol. 2, no. 7, pp. 800-803, 1996.

[76] E. Hu, P. Liang, and B. M. Spiegelman, "AdipoQ is a novel adipose-specific gene dysregulated in obesity," Journal of Biological Chemistry, vol. 271, no. 18, pp. 10697-10703, 1996.

[77] N. Barbarroja, S. Rodriguez-Cuenca, H. Nygren et al., "Increased dihydroceramide/ceramide ratio mediated by defective expression of degs1 impairs adipocyte differentiation and function," Diabetes, vol. 64, no. 4, pp. 1180-1192, 2015.

[78] S. Mitsutake, T. Date, H. Yokota, M. Sugiura, T. Kohama, and Y. Igarashi, "Ceramide kinase deficiency improves diet-induced obesity and insulin resistance," FEBS Letters, vol. 586, no. 9, pp. 1300-1305, 2012.

[79] A. Kihara and Y. Igarashi, "Production and release of sphingosine 1-phosphate and the phosphorylated form of the immunomodulator FTY720," Biochimica et Biophysica Acta, vol. 1781, no. 9, pp. 496-502, 2008.

[80] K. Takabe, S. W. Paugh, S. Milstien, and S. Spiegel, “'Inside-out” signaling of sphingosine-1-phosphate: therapeutic targets," Pharmacological Reviews, vol. 60, no. 2, pp. 181-195, 2008.

[81] M. R. Kendall and C. J. Hupfeld, "FTY720, a sphingosine-1phosphate receptor modulator, reverses high-fat diet-induced weight gain, insulin resistance and adipose tissue inflammation in C57BL/6 mice," Diabetes, Obesity and Metabolism, vol. 10, no. 9, pp. 802-805, 2008.

[82] M.-H. Moon, J.-K. Jeong, J.-H. Lee et al., "Antiobesity activity of a sphingosine 1-phosphate analogue FTY720 observed in adipocytes and obese mouse model," Experimental and Molecular Medicine, vol. 44, no. 10, pp. 603-614, 2012.

[83] T. Hashimoto, J. Igarashi, and H. Kosaka, "Sphingosine kinase is induced in mouse 3T3-L1 cells and promotes adipogenesis," Journal of Lipid Research, vol. 50, no. 4, pp. 602-610, 2009. 
[84] L. F. Van Gaal, I. L. Mertens, and C. E. De Block, "Mechanisms linking obesity with cardiovascular disease," Nature, vol. 444, no. 7121, pp. 875-880, 2006.

[85] J. E. Deanfield, J. P. Halcox, and T. J. Rabelink, "Endothelial function and dysfunction: testing and clinical relevance," Circulation, vol. 115, no. 10, pp. 1285-1295, 2007.

[86] M. Chakraborty, C. Lou, C. Huan et al., "Myeloid cell-specific serine palmitoyltransferase subunit 2 haploinsufficiency reduces murine atherosclerosis," Journal of Clinical Investigation, vol. 123, no. 4, pp. 1784-1797, 2013.

[87] T.-S. Park, R. L. Panek, S. B. Mueller et al., "Inhibition of sphingomyelin synthesis reduces atherogenesis in apolipoprotein E-knockout mice," Circulation, vol. 110, no. 22, pp. 3465-3471, 2004.

[88] Q.-J. Zhang, W. L. Holland, L. Wilson et al., "Ceramide mediates vascular dysfunction in diet-induced obesity by PP2Amediated dephosphorylation of the eNOS-Akt complex," Diabetes, vol. 61, no. 7, pp. 1848-1859, 2012.

[89] V. C. Mehra, E. Jackson, X. M. Zhang et al., "Ceramideactivated phosphatase mediates fatty acid-induced endothelial VEGF resistance and impaired angiogenesis," American Journal of Pathology, vol. 184, no. 5, pp. 1562-1576, 2014.

[90] S. L. Schissel, J. Tweedie-Hardman, J. H. Rapp, G. Graham, K. J. Williams, and I. Tabas, "Rabbit aorta and human atherosclerotic lesions hydrolyze the sphingomyelin of retained low-density lipoprotein. Proposed role for arterial-wall sphingomyelinase in subendothelial retention and aggregation of atherogenic lipoproteins," Journal of Clinical Investigation, vol. 98, no. 6, pp. 1455-1464, 1996.

[91] Y.-R. Zhao, J.-B. Dong, Y. Li, and M.-P. Wu, "Sphingomyelin synthase 2 over-expression induces expression of aortic inflammatory biomarkers and decreases circulating EPCs in ApoE KO mice," Life Sciences, vol. 90, no. 21-22, pp. 867-873, 2012.

[92] M. R. Hojjati, Z. Li, H. Zhou et al., "Effect of myriocin on plasma sphingolipid metabolism and atherosclerosis in apoE-deficient mice," Journal of Biological Chemistry, vol. 280, no. 11, pp. 1028410289, 2005.

[93] E. N. Glaros, W. S. Kim, B. J. Wu et al., "Inhibition of atherosclerosis by the serine palmitoyl transferase inhibitor myriocin is associated with reduced plasma glycosphingolipid concentration," Biochemical Pharmacology, vol. 73, no. 9, pp. 1340-1346, 2007.

[94] B. Garner, H. R. Mellor, T. D. Butters, R. A. Dwek, and F. M. Platt, "Modulation of THP-1 macrophage and cholesterolloaded foam cell apolipoprotein E levels by glycosphingolipids," Biochemical and Biophysical Research Communications, vol. 290, no. 5, pp. 1361-1367, 2002.

[95] N. Gong, H. Wei, S. H. Chowdhury, and S. Chatterjee, "Lactosylceramide recruits $\mathrm{PKC} \alpha / \varepsilon$ and phospholipase $\mathrm{A}_{2}$ to stimulate PECAM-1 expression in human monocytes and adhesion to endothelial cells," Proceedings of the National Academy of Sciences of the United States of America, vol. 101, no. 17, pp. 64906495, 2004.

[96] S. Chatterjee, D. Bedja, S. Mishra et al., "Inhibition of glycosphingolipid synthesis ameliorates atherosclerosis and arterial stiffness in apolipoprotein $\mathrm{E}^{-/-}$mice and rabbits fed a high-fat and -cholesterol diet," Circulation, vol. 129, no. 23, pp. 2403-2413, 2014.

[97] E. N. Glaros, W. S. Kim, K.-A. Rye, J. A. Shayman, and B. Garner, "Reduction of plasma glycosphingolipid levels has no impact on atherosclerosis in apolipoprotein E-null mice," Journal of Lipid Research, vol. 49, no. 8, pp. 1677-1681, 2008.
[98] C. J. Knowles, M. Cebova, and I. M. Pinz, "Palmitate dietinduced loss of cardiac caveolin-3: a novel mechanism for lipid-induced contractile dysfunction," PLoS ONE, vol. 8, no. 4, Article ID e61369, 2013. 


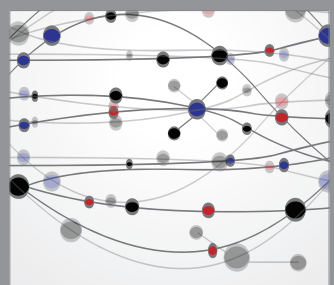

The Scientific World Journal
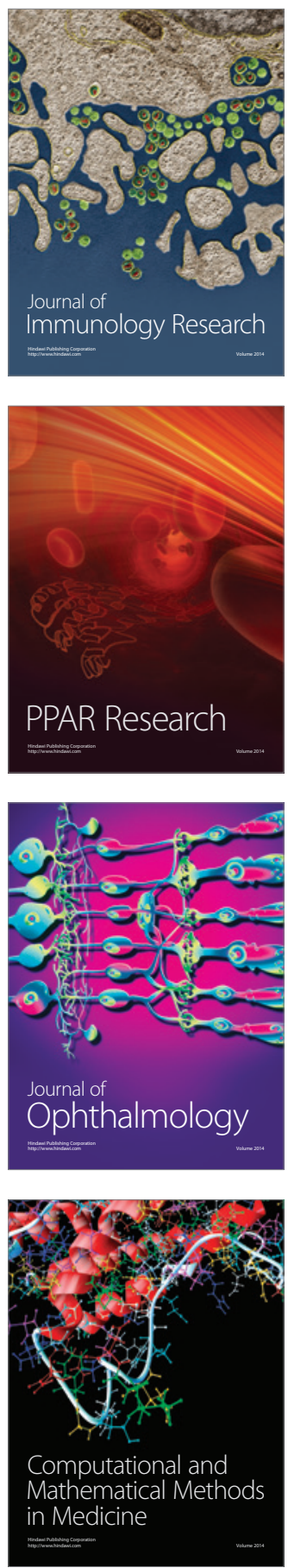

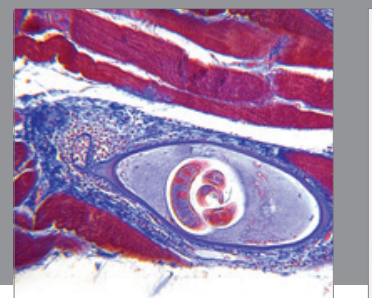

Gastroenterology

Research and Practice
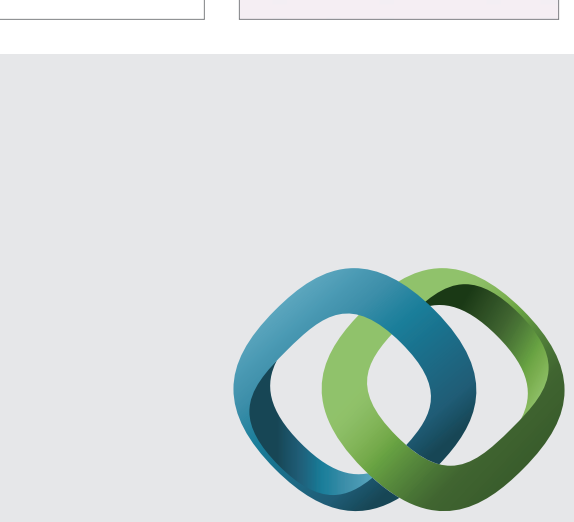

\section{Hindawi}

Submit your manuscripts at

http://www.hindawi.com
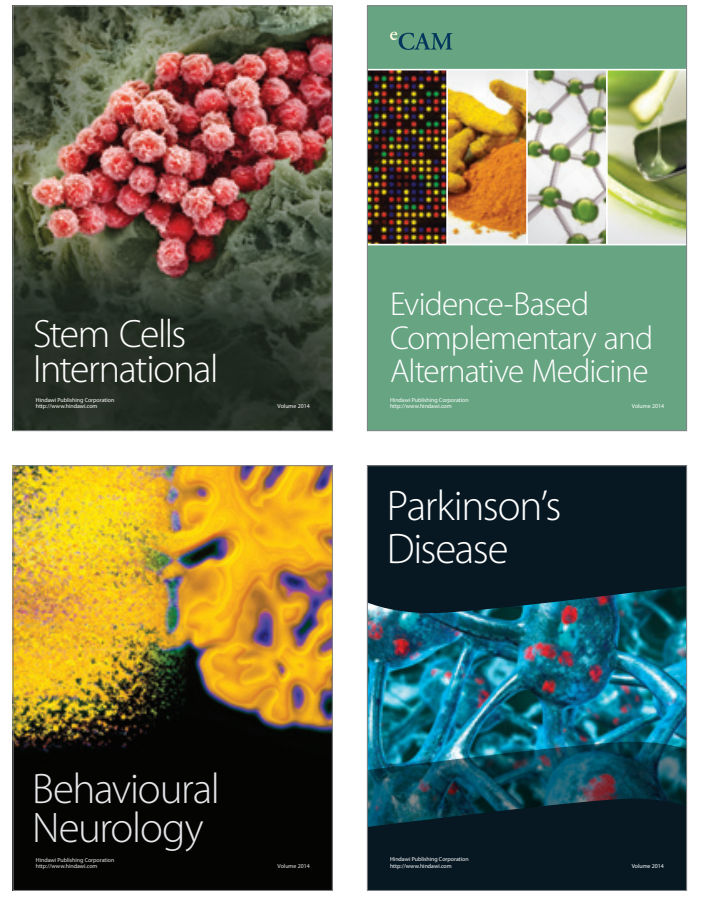
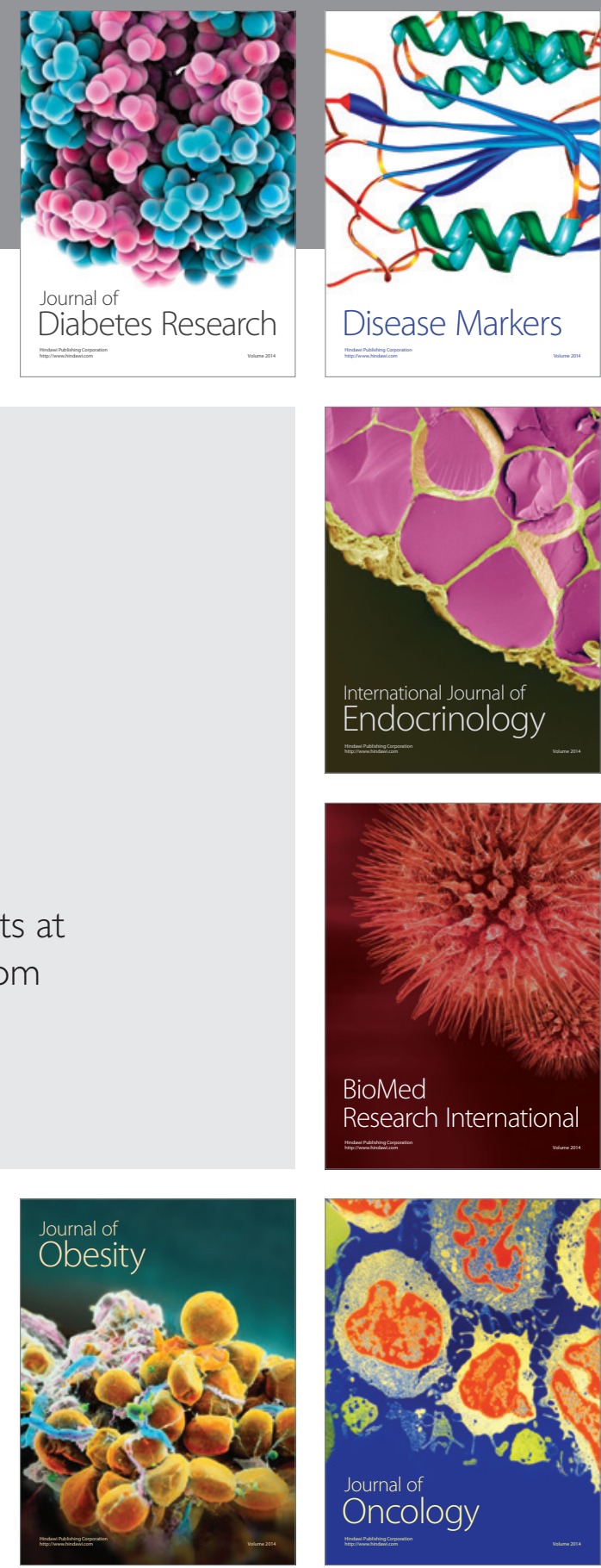

Disease Markers
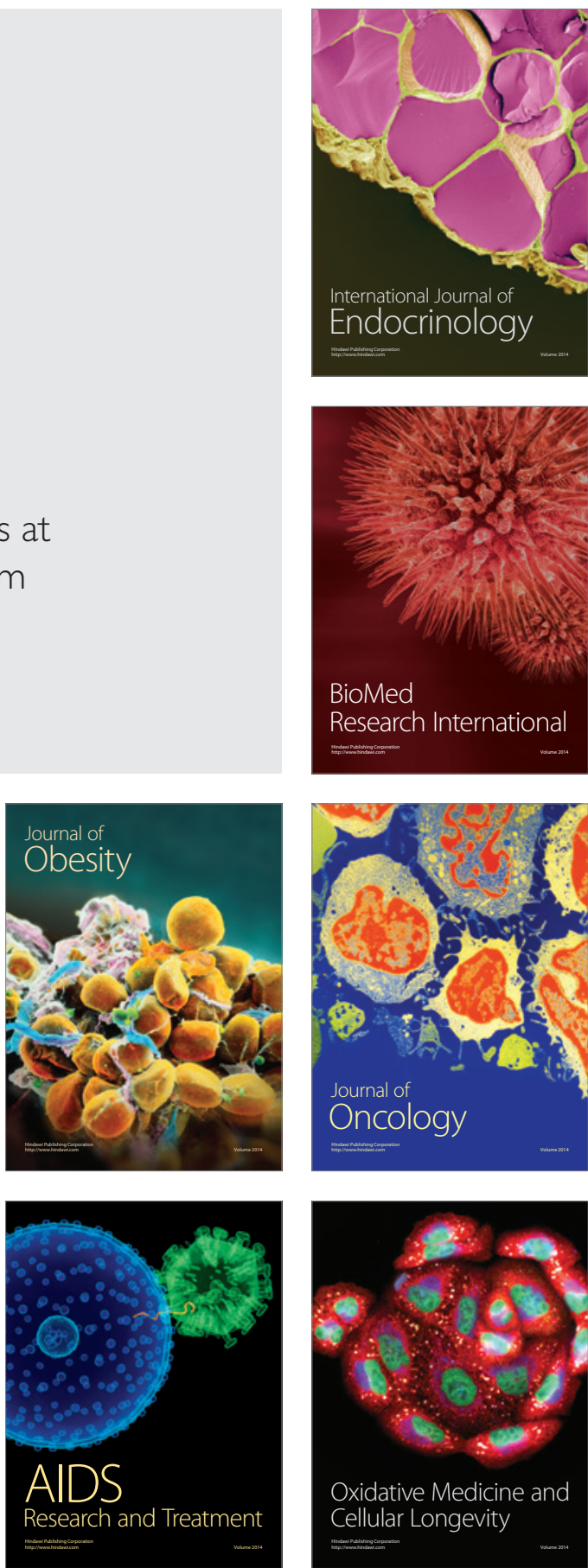